\title{
Birth of microbubbles in turbulent breaking waves
}

\author{
Wai Hong Ronald Chan $\odot,{ }^{*}$ Shahab Mirjalili, ${ }^{\dagger}$ Suhas S. Jain, Javier Urzay, \\ Ali Mani, and Parviz Moin \\ Center for Turbulence Research, Stanford University, Stanford, California 94305, USA
}

(Received 28 January 2019; published 24 October 2019)

\begin{abstract}
This paper is associated with a video winner of a 2018 APS/DFD Gallery of Fluid Motion Award for work presented at the DFD Gallery of Fluid Motion. The original video is available online at the Gallery of Fluid Motion, https://doi.org/10.1103/APS.DFD.2018. GFM.V0027
\end{abstract}

DOI: 10.1103/PhysRevFluids.4.100508

Microbubbles have been observed in large-scale two-phase flows like breaking waves [1,2], seafaring vessel wakes $[3,4]$, and raindrops impacting liquid pools [5,6]. These microbubbles rise slowly [3,7] and reside for a long time in oceans, thereby influencing interfacial transport $[1,8]$, surface reflectance $[4,9,10]$, and acoustic wave propagation $[3,11]$. Researchers have measured the distribution of bubble sizes in laboratory breaking waves, including Deane and Stokes [2] and Blenkinsopp and Chaplin [12], who measured bubbles with sizes of $0.1-10 \mathrm{~mm}$. Microbubbles with sizes of 10-100 $\mu \mathrm{m}$, however, were difficult to characterize due to resolution limitations [13]. These microbubbles are hypothesized to form when liquid interfaces collide and entrap thin gas films, which subsequently rupture and retract. Here, the stages involved in this postulated mechanism are outlined, from large to progressively smaller scales.

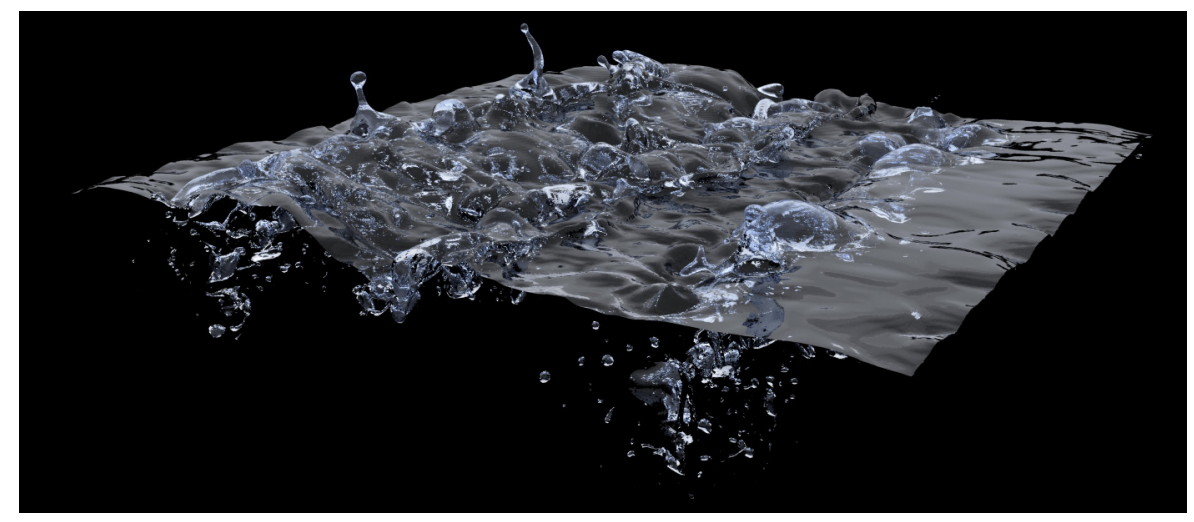

FIG. 1. Snapshot of the side view of the simulated breaking wave, shortly after the wave has broken.

\footnotetext{
*whrchan@stanford.edu

†ssmirjal@stanford.edu
}

Published by the American Physical Society under the terms of the Creative Commons Attribution 4.0 International license. Further distribution of this work must maintain attribution to the author(s) and the published article's title, journal citation, and DOI. 

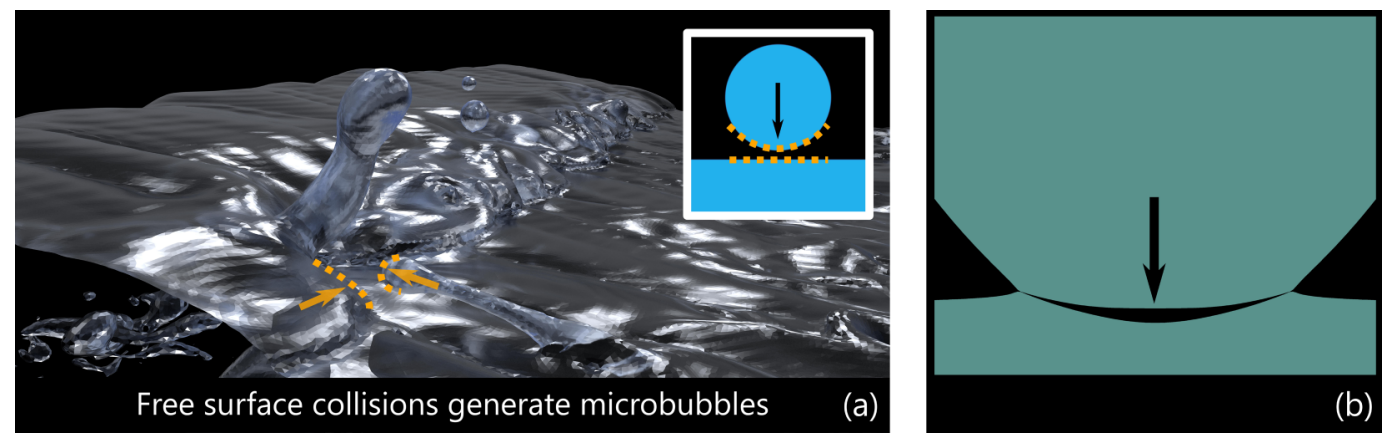

FIG. 2. (a) Close-up view of an imminent collision between interfaces after the wave has broken. Inset: Schematic illustrating the drop-pool model problem. The imminent collision (dashed lines, main image) may be mapped to drop impact on a deep liquid pool (dashed lines, inset). (b) Simulation snapshot depicting drop-pool impact and the ensuing air film entrapment.

To investigate the large scales, a geometric volume-of-fluid-based incompressible two-phase solver with consistent mass and momentum advection $[14,15]$ was used to simulate a $27-\mathrm{cm}$-long breaking Stokes water wave in air $[16,17]$ with a minimum grid size of $0.6 \mathrm{~mm}$, as depicted in Fig. 1 . The simulation revealed that turbulent breaking waves entrain large gas cavities that break up into smaller bubbles. Collisions between interfaces that may trap thin gas films and generate microbubbles were also observed. These films and microbubbles cannot be resolved by this simulation.

The impact of a drop on a deep liquid pool is a suitable model problem for studying how collisions between two arbitrarily curved interfaces may lead to microbubble entrainment, as suggested in Fig. 2(a). This is motivated by the observation of Mesler entrainment of microbubbles in droppool impact experiments [18-20], which revealed that these microbubbles are remnants of a thin gas film sandwiched between the colliding interfaces. Simulations employing the boundary integral method (similar to [21,22]) were performed to capture the film geometry. A simulated water-air impact event with an impact velocity of $0.5 \mathrm{~m} / \mathrm{s}$ and a drop diameter of $1.3 \mathrm{~mm}$ is depicted in Fig. 2(b).

Experiments [20] have shown that once the entrapped gas film ruptures and retracts, transverse edge instabilities will trigger microbubble shedding. Three-dimensional simulations of retracting thin gas films performed using a conservative diffuse interface method developed by Mirjalili et al. [23] also capture this. Retraction of a $2-\mu \mathrm{m}$-thick air film in water is shown in Fig. 3. Microbubbles with diameters of 30-40 $\mu \mathrm{m}$ were shed due to these instabilities [24].

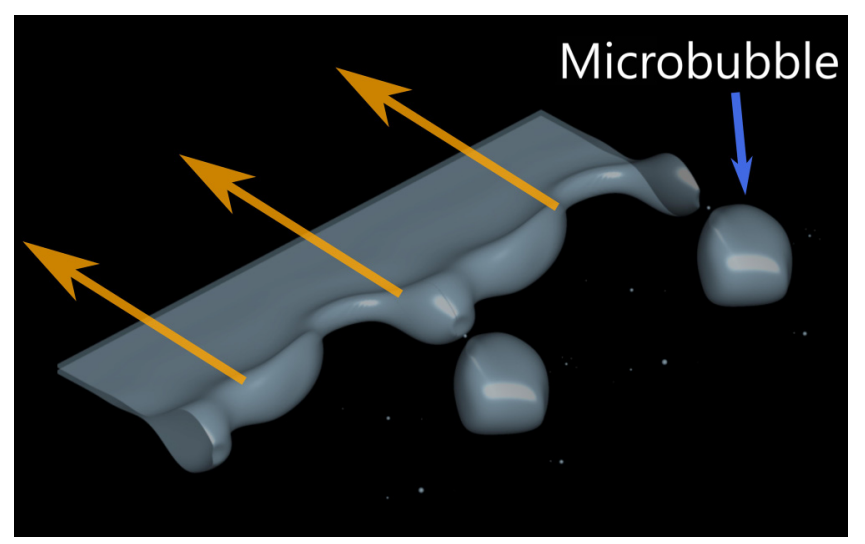

FIG. 3. Simulation snapshot depicting the formation of microbubbles due to the retraction of a thin air film. The large arrows indicate the direction of retraction of the rim of the film. 


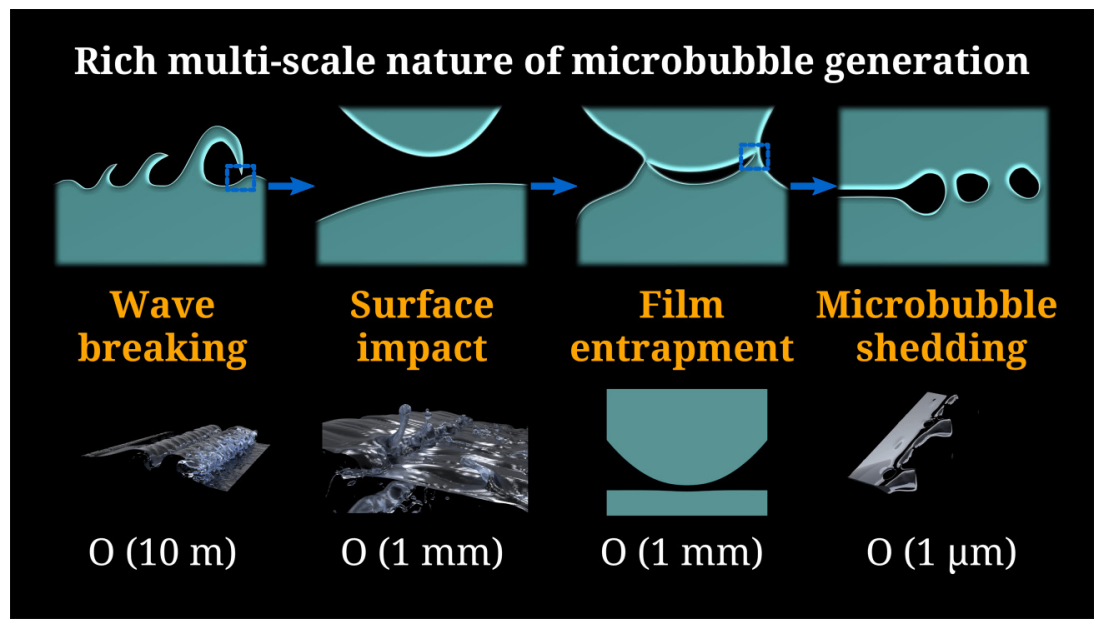

FIG. 4. Schematic describing the various stages in the potential pathway for microbubble formation in breaking waves described in this work, along with the characteristic length scales associated with each stage. The top row comprises sketches of each stage, while the bottom row comprises snapshots of simulations that were performed in this work.

This work outlines a potential pathway for microbubble formation in breaking waves, which is summarized in Fig. 4. Preliminary application of a collision detection algorithm [16] to the wave simulation suggests up to hundreds of microbubble-producing collisions may occur every second, thus creating up to a million microbubbles per cubic meter every second. The sheer number of expected microbubbles, which cannot be resolved in large-scale simulations and require additional modeling, motivates further study of their formation and evolution.

The authors are grateful to M. Bassenne and A. Lozano-Durán for their feedback. W.H.R.C. is supported by a National Science Scholarship from A*STAR, Singapore. S.S.J. is supported by a Franklin P. and Caroline M. Johnson Fellowship from Stanford University. W.H.R.C., S.S.J., J.U., and P.M. acknowledge support from ONR, Grant No. N00014-15-1-2726. S.M. and A.M. acknowledge support from ONR, Grant No. N00014-15-1-2523. Computational resources from DOE and the Certainty Cluster awarded by NSF to CTR are also acknowledged.

[1] W. K. Melville, The role of surface-wave breaking in air-sea interaction, Annu. Rev. Fluid Mech. 28, 279 (1996).

[2] G. B. Deane and M. D. Stokes, Scale dependence of bubble creation mechanisms in breaking waves, Nature (London) 418, 839 (2002).

[3] M. V. Trevorrow, S. Vagle, and D. M. Farmer, Acoustical measurements of microbubbles within ship wakes, J. Acoust. Soc. Am. 95, 1922 (1994).

[4] A. M. Reed and J. H. Milgram, Ship wakes and their radar images, Annu. Rev. Fluid Mech. 34, 469 (2002).

[5] D. C. Blanchard and A. H. Woodcock, Bubble formation and modification in the sea and its meteorological significance, Tellus 9, 145 (1957).

[6] A. Prosperetti and H. N. Õguz, The impact of drops on liquid surfaces and the underwater noise of rain, Annu. Rev. Fluid Mech. 25, 577 (1993). 
[7] S. A. Thorpe, On the clouds of bubbles formed by breaking wind-waves in deep water, and their role in air-sea gas transfer, Philos. Trans. R. Soc. London, Ser. A 304, 155 (1982).

[8] S. A. Thorpe, Bubble clouds and the dynamics of the upper ocean, Q. J. R. Meteorol. Soc. 118, 1 (1992).

[9] E. J. Terrill, W. K. Melville, and D. Stramski, Bubble entrainment by breaking waves and their influence on optical scattering in the upper ocean, J. Geophys. Res. 106, 16815 (2001).

[10] X. Zhang, M. Lewis, W. P. Bissett, B. Johnson, and D. Kohler, Optical influence of ship wakes, Appl. Opt. 43, 3122 (2004).

[11] H. C. Pumphrey and L. A. Crum, Acoustic emissions associated with drop impacts, in Sea Surface Sound: Natural Mechanisms of Surface-Generated Noise in the Ocean, edited by B. R. Kerman (Kluwer Academic, Dordrecht, 1988), pp. 463-483.

[12] C. E. Blenkinsopp and J. R. Chaplin, Bubble size measurements in breaking waves using optical fiber phase detection probes, IEEE J. Ocean Eng. 35, 388 (2010).

[13] Microbubbles smaller than $10 \mu \mathrm{m}$ generally dissolve completely before reaching the surface [3,7] and will not be considered.

[14] F. Ham, D. Kim, S. Bose, H. Le, and M. Herrmann, Simulation of liquid fuel atomization by a complex high-shear swirling injector, Proceedings of the ASME Turbo Expo 2014: Turbine Technical Conference and Exposition. Volume 4B: Combustion, Fuels and Emissions, Düsseldorf, Germany (2014), doi: 10.1115/GT2014-27220.

[15] L. Bravo, D. Kim, F. Ham, and S. Su, Computational study of atomization and fuel drop size distributions in high-speed primary breakup, Atomization and Sprays 28, 321 (2018).

[16] W. H. R. Chan, J. Urzay, and P. Moin, Subgrid-scale modeling for microbubble generation amid colliding water surfaces, in Proceedings of the 32nd Symposium on Naval Hydrodynamics, Hamburg, Germany (2018), arXiv:1811.11898.

[17] W. H. R. Chan, M. S. Dodd, P. L. Johnson, J. Urzay, and P. Moin, Formation and dynamics of bubbles in breaking waves: Part II. The evolution of the bubble size distribution and breakup/coalescence statistics, in Annual Research Briefs (Center for Turbulence Research, Stanford University, 2018), pp. 21-34.

[18] L. Esmailizadeh and R. Mesler, Bubble entrainment with drops, J. Colloid Interface Sci. 110, 561 (1986).

[19] S. T. Thoroddsen, T. G. Etoh, and K. Takehara, Air entrapment under an impacting drop, J. Fluid Mech. 478, 125 (2003).

[20] S. T. Thoroddsen, M.-J. Thoraval, K. Takehara, and T. G. Etoh, Micro-bubble morphologies following drop impacts onto a pool surface, J. Fluid Mech. 708, 469 (2012).

[21] M. Mani, S. Mandre, and M. P. Brenner, Events before droplet splashing on a solid surface, J. Fluid Mech. 647, 163 (2010).

[22] P. D. Hicks and R. Purvis, Air cushioning in droplet impacts with liquid layers and other droplets, Phys. Fluids 23, 062104 (2011).

[23] S. Mirjalili, C. B. Ivey, and A. Mani, A conservative diffuse interface method for two-phase flows with provable boundedness properties, arXiv: 1803.01262.

[24] S. Mirjalili, W. H. R. Chan, and A. Mani, High fidelity simulations of micro-bubble shedding from retracting thin gas films in the context of liquid-liquid impact, in Proceedings of the 32nd Symposium on Naval Hydrodynamics, Hamburg, Germany (2018), arXiv:1811.12352. 